\title{
Chemokine receptor mutant CX3CR1-M280 has impaired adhesive function and correlates with protection from cardiovascular disease in humans
}

\author{
David H. McDermott, ${ }^{1}$ Alan M. Fong, ${ }^{2}$ Qiong Yang, ${ }^{3}$ Joan M. Sechler, ${ }^{1}$ \\ L. Adrienne Cupples, ${ }^{3}$ Maya N. Merrell, ${ }^{1}$ Peter W.F. Wilson, ${ }^{4}$ Ralph B. D’Agostino, ${ }^{5}$ \\ Christopher J. O’Donnell, ${ }^{6,7}$ Dhavalkumar D. Patel, ${ }^{2}$ and Philip M. Murphy ${ }^{1}$
}

\author{
${ }^{1}$ Laboratory of Host Defenses, National Institute of Allergy and Infectious Diseases, NIH, Bethesda, Maryland, USA \\ ${ }^{2}$ Department of Medicine, Duke University Medical Center, Durham, North Carolina, USA \\ ${ }^{3}$ Department of Biostatistics, School of Public Health, \\ ${ }^{4}$ School of Medicine, and \\ ${ }^{5}$ Department of Mathematics, Boston University, Boston, Massachusetts, USA \\ ${ }^{6}$ National Heart, Lung, and Blood Institute, NIH, Bethesda, Maryland, USA \\ ${ }^{7}$ Framingham Heart Study, Framingham, Massachusetts, USA
}

\begin{abstract}
The chemokine receptor CX3CR1 is a proinflammatory leukocyte receptor specific for the chemokine fractalkine (FKN or CX3CL1). In two retrospective studies, CX3CR1 has been implicated in the pathogenesis of atherosclerotic cardiovascular disease (CVD) based on statistical association of a common receptor variant named CX3CR1-M280 with lower prevalence of atherosclerosis, coronary endothelial dysfunction, and acute coronary syndromes. However, the general significance of CX3CR1-M280 and its putative mechanism of action have not previously been defined. Here we show that FKN-dependent cellcell adhesion under conditions of physiologic shear is severely reduced in cells expressing CX3CR1-M280. This was associated with marked reduction in the kinetics of FKN binding as well as reduced FKN-induced chemotaxis of primary leukocytes from donors homozygous for CX3CR1-M280. We also show that CX3CR1$M 280$ is independently associated with a lower risk of CVD (adjusted odds ratio, $0.60, P=0.008$ ) in the Offspring Cohort of the Framingham Heart Study, a long-term prospective study of the risks and natural history of this disease. These data provide mechanism-based and consistent epidemiologic evidence that CX3CR1 may be involved in the pathogenesis of CVD in humans, possibly by supporting leukocyte entry into the coronary artery wall. Moreover, they suggest that CX3CR1-M280 is a genetic risk factor for CVD.
\end{abstract}

J. Clin. Invest. 111:1241-1250 (2003). doi:10.1172/JCI200316790.

\section{Introduction}

Despite advances in prevention and treatment, atherosclerotic cardiovascular disease (CVD) remains the leading cause of mortality in the United States (1). Although well-established risk factors such as elevated serum cholesterol, high blood pressure, diabetes, smoking, male sex, and obesity have been identified, these factors do not fully account for an individual's risk of CVD (2). A family history of premature CVD is also an independ-

Received for publication August 29, 2002, and accepted in revised form January 21, 2003.

Address correspondence to: Philip Murphy, Laboratory of Host Defenses, National Institute of Allergy and Infectious Diseases, NIH, Building 10, Room 11N113, 9000 Rockville Pike, Bethesda, Maryland 20892-1886, USA. Phone: (301) 496-8616;

Fax: (301) 402-4369; E-mail: pmm@nih.gov.

Conflict of interest: The authors have declared that no conflict of interest exists.

Nonstandard abbreviations used: cardiovascular disease (CVD); fractalkine (FKN); Accidents Coronaires Aigus Bichat (ACABI); wild-type CX3CR1 (CX3CR1-WT); Framingham Heart Study (FHS); odds ratio (OR). ent risk factor, implying that genetic factors in addition to those linked to the above risk factors also play an important role (3). Identifying these factors is likely to lead to a better understanding of pathogenesis and to improved treatment and prevention strategies.

A cardinal feature of atherosclerosis is inflammation of the vessel wall arising from interactions of leukocytes with vascular endothelial cells, smooth muscle cells, and fibroblasts (4-6). At the molecular level, interactions among these cell types are regulated by cytokines, adhesion molecules, and chemoattractants. Chemokines are a large family of chemoattractants that direct migration of leukocytes from the blood to sites of inflammation $(7,8)$. Moreover, chemokines such as MCP-1 (CCL2), RANTES (CCL5), SDF-1 (CXCL12), and fractalkine (FKN, or CX3CL1 in the standard chemokine nomenclature) have been identified in human atherosclerotic lesions (9-12), and mice genetically deficient in MCP-1 and its receptor CCR2 have reduced susceptibility to atherosclerosis (13-15). Nevertheless, the importance of specific chemokines in 
CVD has not yet been established in humans. Recently, based on expression, functional, and epidemiologic data, FKN and its receptor, CX3CR1, have emerged as particularly interesting candidates $(11,12,16-19)$.

FKN is an unusual chemokine because in addition to a classic chemokine domain, it has a mucin-like stalk, a transmembrane domain, and a cytoplasmic tail allowing the molecule to be tethered to cells that express it, which include inflamed endothelial cells $(20,21)$. Even under conditions of physiologic flow, FKN can mediate strong, direct, and rapid adherence of leukocytes expressing CX3CR1, which include most NK cells and monocytes as well as subpopulations of both $\mathrm{CD}^{+}$and $\mathrm{CD}^{+}$cytotoxic T lymphocytes $(16,22,23)$. Importantly, FKNdependent adhesion of NK cells to endothelium has been shown to promote endothelial cell damage (24). Additionally, FKN may be cleaved by TNF- $\alpha$-converting enzyme (TACE) and released from cells $(25,26)$. In this form it is able to act as a typical leukocyte chemoattractant at CX3CR1 via the classic $\mathrm{G}_{\mathrm{i}}$-coupled signaling pathway common to almost all chemokines $(16,22,23)$. Although the adhesive activity of CX3CR1 does not require activation of $\mathrm{G}_{\mathrm{i}}$-signaling, signaling does upregulate cell adhesion to fibronectin and ICAM-1 (27).

We have recently identified two nonsynonymous single-nucleotide polymorphisms affecting codons 249 and 280 of CX3CR1 that are in complete linkage disequilibrium and that were both associated with reduced prevalence of atherosclerosis and coronary endothelial dysfunction in an NIH cardiac catheterization cohort and with reduced prevalence of acute coronary events (myocardial infarction and unstable angina) in the Accidents Coronaires Aigus Bichat (ACABI) cohort from France $(18,19)$. The polymorphisms cause amino acid changes from valine to isoleucine at codon 249 (V249I) and from threonine to methionine at position 280 (T280M); these codons are in helices 6 and 7, respectively, of the seven-transmembrane domain receptor architecture (28). Since CX3CR1-M280 always occurs in the presence of CX3CR1-I249, we will refer to the receptor protein bearing both of these amino acids as CX3CR1M280. Based on homology with the known structure of rhodopsin, these amino acids are both located in the portion of the helices near the outside the cell (28).

Although our previous reports about CX3CR1-M280 in CVD were consistent with each other, they were based on small case-control studies and therefore could be significantly influenced by selection bias $(18,19)$. In addition, we did not establish a causal mechanism of action in CVD since no major defect in recognition of FKN was identified for this variant. To further test the relationship of CX3CR1 to the pathogenesis of CVD, we have now addressed both of these issues directly.

\section{Methods}

Preparation of stably transfected cell lines. HEK 293 and K562 cell lines from American Type Culture Collection (Manassas, Virginia, USA) were electroporated with pcDNA3.1 expression plasmids (Invitrogen Corp.,
Carlsbad, California, USA) encoding wild-type CX3CR1 (CX3CR1-WT) or CX3CR1-M280 (16). Plasmid inserts were derived from genomic DNA by high-fidelity PCR amplification (Invitrogen Corp.) and were sequenced bidirectionally. After G418 selection for at least 3 weeks, stable receptor expression was verified by flow cytometry. FKN-expressing human endothelial cells (926-FKN cells) were obtained as previously described (29).

FKN binding assays. HEK 293 cells were washed and placed in binding buffer (HBSS containing 1\% BSA and $0.01 \%$ sodium azide). ${ }^{125} \mathrm{I}-\mathrm{FKN}(2,200 \mathrm{Ci} / \mathrm{mmol})$, obtained from PerkinElmer Life Sciences (Shelton, Connecticut, USA), was added to a final concentration of $0.25 \mathrm{nM}$ in $200 \mu \mathrm{l}$ with $10^{5}$ cells at $37^{\circ} \mathrm{C}$ with gentle mixing. For competition binding experiments, unlabeled FKN at various concentrations was added simultaneously with ${ }^{125}$ I-FKN and incubated for 2 hours. For kinetic binding studies, $0.25 \mathrm{nM}{ }^{125} \mathrm{I}-\mathrm{FKN}$ was added to $2 \mathrm{ml}$ of cells at $10^{6} / \mathrm{ml}$. At various timepoints, $10^{5}$ cells $(100 \mu \mathrm{l})$ were removed for determination of cell-associated radioactivity. After 48 minutes, 1,000fold excess unlabeled FKN (250 nM) was added to assess the reversibility of binding. Cells were washed in high-salt stop buffer (binding buffer containing $0.5 \mathrm{M}$ sodium chloride) to remove nonspecifically bound radioligand. Binding to untransfected cells was less than $1 \%$ of total cpm added in all experiments. Each condition was tested in triplicate.

Flow cytometry. PBMCs were isolated using Lymphocyte Separation Medium (Cellgro; Mediatech Inc., Kansas City, Missouri, USA) from the heparinized blood of healthy donors and were kept at room temperature for no more than 30 minutes before use. Washed HEK 293 cells, K562 cells, or PBMCs were stained at $4^{\circ} \mathrm{C}$ for 30 minutes in labeling buffer (HBSS with $0.1 \% \mathrm{BSA}$ and $0.1 \%$ sodium azide) containing antiFc reagent (Miltenyi Biotec, Auburn, California, USA) with directly labeled mAb's or isotype controls (BD Pharmingen, San Diego, California, USA). Human CX3CR1-directed mAb 2A9 was obtained as an FITC conjugate from MBL International Corp. (Nagoya, Japan). In some experiments, unconjugated mAb 2A9 (a generous gift from Toshio Imai of the Kan Research Institute, Kyoto, Japan) was used instead, with secondary detection by a goat anti-rat phycoerythrin-conjugated polyclonal $\mathrm{Ab}$ (Caltag Laboratories Inc., Burlingame, California, USA). Flow cytometry was performed with a FACScan and data were analyzed using CellQuest software (Becton, Dickinson and Co., San Jose, California, USA) after gating of dead cells using propidium iodide and correction of results for nonspecific staining using isotype antibody controls.

Parallel plate flow chamber adhesion assay. Experiments to determine the interaction between CX3CR1 variants and FKN were carried out as previously described (16) and recorded on videotape. Briefly, 926 cells or 926FKN-transfected cells were plated onto glass coverslips in a six-well cluster dish the day before the experiment and allowed to grow to confluence. Stably transfected 
K562 cells (expressing either CX3CR1-WT or CX3CR1M280) or untransfected K562 cells were resuspended in flow buffer (PBS containing $0.75 \mathrm{mM} \mathrm{CaCl}_{2}, 0.75 \mathrm{mM}$ $\mathrm{MgCl}_{2}$, and $0.5 \% \mathrm{BSA}$ ) at a concentration of $10^{6} \mathrm{cells} / \mathrm{ml}$. The cells were loaded and allowed to interact with 926 cells in the flow chamber at room temperature or $37^{\circ} \mathrm{C}$ at a shear stress of 0.25 dynes $/ \mathrm{cm}^{2}$ for 5 minutes. The shear stresses were adjusted using a Harvard model 44 syringe pump (Harvard Apparatus Co., South Natick, Massachusetts, USA). Following 1-minute washes at 0.5, $1,1.85$, and 5 dynes $/ \mathrm{cm}^{2}$, cells were subjected to a shear stress of 10 dynes $/ \mathrm{cm}^{2}$ for 5 minutes. Cells remaining firmly attached to the coverslip at the end of this period were counted. Each condition was tested in triplicate.

Calcium flux. Cells were washed and resuspended at $10^{7} / \mathrm{ml}$ in HBSS containing $10 \mathrm{mM}$ HEPES prior to the addition of $2.5 \mu \mathrm{M}$ fura-2 AM (Molecular Probes Inc., Eugene, Oregon, USA). For PBMCs, $1.5 \mu \mathrm{l}$ of a $20 \%$ DMSO solution of Pluronic F-127 (Molecular Probes Inc.) per $10^{7}$ cells was mixed with fura-2 AM to HEK 293, or the same amount of fura-2 AM mixed 1:1 with a 20\% DMSO solution of Pluronic F-127 (Molecular Probes Inc., Eugene, Oregon, USA) for PBMC. Cells were incubated at $37^{\circ} \mathrm{C}$ for 45 minutes, washed once, incubated at $25^{\circ} \mathrm{C}$ for 45 minutes, washed, and then resuspended in HBSS. Cells $\left(2 \times 10^{6}\right.$ in $\left.100 \mu \mathrm{l}\right)$ were pipetted into each well of a 96-well plate (Greiner Bioone Inc., Longwood, Florida, USA) coated with poly-Llysine (Sigma-Aldrich, St. Louis, Missouri, USA) and centrifuged at $400 \mathrm{~g}$ for 10 minutes. The plate was then loaded into the reading chamber of a FlexStation (Molecular Devices Corp., Sunnyvale, California, USA) set at $37^{\circ} \mathrm{C}$ and chemokines were added robotically. Fluorescence intensities emitted by cells excited at 340 and 380 $\mathrm{nm}$ were monitored at $510 \mathrm{~nm}$. Data are the average of triplicates expressed as change in relative fluorescence calculated as the ratio of the two intensities using SoftmaxPro Software (Molecular Devices Corp.), subtracting the baseline from the peak change. Any change with buffer alone was also subtracted.

Chemotaxis. Chemotaxis was performed using a previously published method (30). Briefly, PBMCs isolated as above were immunophenotyped and placed in chemotaxis buffer (RPMI containing $1 \%$ BSA and 10 $\mathrm{mM}$ HEPES). Cells $\left(5 \times 10^{5}\right.$ in $\left.100 \mu \mathrm{l}\right)$ were loaded into the top of each well of a Transwell chemotaxis plate (Corning-Costar Corp., Cambridge, Massachusetts, USA) with $5-\mu \mathrm{m}$ pore inserts. The bottom of each well contained $600 \mu \mathrm{l}$ of prewarmed chemotaxis buffer with the indicated chemokine concentration. SDF-1 $\alpha$ and the chemokine domain of FKN were obtained from PeproTech Inc. (Rocky Hill, New Jersey, USA). The plate was then incubated for 90 minutes at $37^{\circ} \mathrm{C}$ in a $5 \% \mathrm{CO}_{2}$ atmosphere, and cells migrating to the lower chamber were counted and immunophenotyped. Specific chemotaxis is expressed as the percentage of cells that migrated (no. migrated/no. loaded), subtracting the response to buffer alone. All conditions were run in triplicate.
Framingham Heart Study Offspring Cohort subjects. The design and methodology of the Framingham Heart Study (FHS) has been previously published $(31,32)$. The Offspring Cohort of the FHS enrolled 5,124 children of the original participants and their spouses beginning in the year 1971 . They undergo a comprehensive medical exam every 4 years (defined as a cycle). The FHS Offspring Cohort contained 1,888 unrelated participants for whom DNA was available. Of these, 1,861 had given informed consent for genotyping and were entered into the present study. All subjects are Caucasian and all but two samples yielded informative genotyping results. The study was approved by the Institutional Review Boards of the National Institute of Allergy and Infectious Diseases and Boston University School of Medicine.

Clinical definitions. CVD and established CVD risk factors were evaluated for the Offspring Study by a threephysician endpoint committee at the time of the sixth cycle (i.e., including data up to the year 1997, the last year for which data are complete) (33). Of the 1,859 subjects whose genotypes were determined, 204 $(11.0 \%)$ met the definition for CVD at the time of the blood draw. Prevalent CVD was defined as a prior diagnosis of coronary heart disease (cardiac death, angina, or myocardial infarction; $n=149$, or $73 \%$ of cases), cerebrovascular disease (stroke or transient ischemic attack; $n=39$, or $19 \%$ of cases), or peripheral vascular disease (intermittent claudication; $n=47$, or $23 \%$ of cases). Note that the numbers add to more than $100 \%$ because of overlap of diagnoses, especially coronary heart disease and intermittent claudication, for which 19 individuals had both diagnoses. Surveillance for CVD events consisted of regular examinations at the study clinic and review of medical records from physician office visits and hospitalizations for heart and cerebrovascular disease. Smoking was defined as a current or prior history of tobacco use within 24 years of the blood draw. Diabetes was defined as a fasting blood glucose level of greater than $7.8 \mathrm{nmol} / 1$ (140 $\mathrm{mg} / \mathrm{dl}$ ), or treatment with dietary modification, insulin, or oral hypoglycemic agents at the time of the study. Hypertension was defined as a systolic blood pressure of $140 \mathrm{mmHg}$ or higher and/or diastolic pressure of $90 \mathrm{mmHg}$ or higher, or treatment with antihypertensive medication.

Genotyping. CX3CR1 genotyping was performed on coded DNA samples by laboratory personnel who had no knowledge of the clinical status of the participants, using previously published methods (18).

Statistical analysis. Unadjusted univariate analyses of the relationship of CX3CR1 genotypes with prevalent CVD were performed by simple cross-tabulation and calculations of odds ratios (ORs). Multivariable-adjusted logistic regression analyses were conducted to examine the independent contribution of CX3CR1 genotype with prevalent CVD (Table 1). The OR and 95\% confidence limit were calculated using this model, and significance of the associations was evaluated based on $\chi^{2}$ tests (SAS 
Clinical characteristics of participants in the FHS Offspring Cohort

\begin{tabular}{|c|c|c|c|c|}
\hline & $\operatorname{CVD}(n=204)$ & No CVD $(n=1,655)$ & OR & $P$ \\
\hline Age (yr) & $65.9 \pm 8.4$ & $58.8 \pm 9.8$ & $1.97^{\mathrm{A}}$ & $<0.0001$ \\
\hline Alcohol consumption (oz/wk) & $2.4 \pm 3.8$ & $2.4 \pm 3.5$ & 0.96 & 0.10 \\
\hline $\operatorname{BMI}\left(\mathrm{kg} / \mathrm{m}^{2}\right)$ & $28.6 \pm 5.2$ & $27.9 \pm 5.0$ & 1.00 & 0.84 \\
\hline HDL cholesterol (mg/dl) & $44.6 \pm 14.4$ & $51.5 \pm 16.1$ & $0.90^{A}$ & 0.42 \\
\hline Total cholesterol (mg/dl) & $198.3 \pm 47.2$ & $206.3 \pm 40.5$ & 1.00 & 0.69 \\
\hline Triglycerides (mg/dl) & $173.6 \pm 171.5$ & $141.4 \pm 160.6$ & 1.00 & 0.22 \\
\hline Male sex (\%) & 65.9 & 47.8 & 1.64 & 0.02 \\
\hline Hypertension (\%) & 59.1 & 30.0 & 1.05 & 0.79 \\
\hline Diabetes (\%) & 23.2 & 6.0 & 2.05 & 0.003 \\
\hline Current or former smokers (\%) & 82.5 & 64.7 & 2.35 & 0.0002 \\
\hline Use of statins (\%) & 30.8 & 9.6 & 2.18 & 0.0002 \\
\hline Use of $\beta$ blockers (\%) & 47.1 & 10.3 & 5.27 & $<0.0001$ \\
\hline Estrogen therapy (\%, women only) & 33.3 & 34.2 & 1.22 & 0.54 \\
\hline Menopause (\%, women only) & 94.4 & 77.5 & 1.03 & 0.96 \\
\hline
\end{tabular}

${ }^{\mathrm{A} O R}$ is given per 10 years for age and per $10 \mathrm{mg} / \mathrm{dl}$ increase for $\mathrm{HDL}$. OR, odds ratio; oz, ounce. versus CX3CR1-M280, respectively $(n=3)$. We infer from this that mAb 2A9, used to select these two cell lines for similar receptor expression, in fact does bind equivalently to CX3CR1-WT and CX3CR1M280 and therefore can be used to assess relative expression of the two variants.

Since CX3CR1 is an adhesion molecule, its ability to capture cells may depend critically on FKN binding rate, a parameter that may not be revealed by equilibrium competition binding analysis at 2 hours. To directly compare this parameter for CX3CR1WT and CX3CR1-M280, we version 8; The SAS Institute Inc., Cary, North Carolina, USA). Independence between established risk factors and CX3CR1 genotypes was examined by a $\chi^{2}$ test with 1 degree of freedom for dichotomous measurements and by testing the difference in mean of the risk factor between genotype groups using a two-tailed Student $t$ test for continuous measurements. Tests of interaction were also performed using a Breslow-Day test of the association of the CX3CR1-M280 allele with CVD, stratified by age (less than 60 years vs. 60 years or more of age), sex, diabetes, smoking, and use of $\beta$ blockers or statins.

\section{Results}

Defective FKN binding to CX3CR1-M280. We hypothesized that the association of CX3CR1-M280 with reduced risk of atherosclerosis and acute coronary events may be due to reduced ligand-receptor binding. To address this directly, we carried out equilibrium competition binding ( 2 hours at $37^{\circ} \mathrm{C}$ in the presence of azide) using $\mathrm{mAb}$ 2A9 to select separate HEK 293 cell lines with similar surface expression of CX3CR1-WT and CX3CR1-M280, as determined by FACS (Figure 1a). This is the first time FKN binding affinity for CX3CR1-WT and CX3CR1M280 has been directly compared using recombinant receptors. FKN binding was similar for both receptors (Figure $1 \mathrm{~b}$ ). Scatchard analysis gave a $K_{\mathrm{d}}$ of $1.3 \pm 0.2 \mathrm{nM}$ and $2.3 \pm 0.5 \mathrm{nM}$ for CX3CR1-WT and CX3CR1-M280, respectively $(n=3)$. These values are close to the value previously published for CX3CR1-WT in transfected HEK 293 cells binding at room temperature (34). Moatti et al. and Faure et al. have previously published two studies indicating that FKN binds to PBMCs with higher affinity than this (Kd = 10-250 pM); however, consistent with the present results, only small differences were observed for CX3CR1-M280 homozygotes versus CX3CR1-WT homozygotes $(19,35)$.

The number of binding sites per cell, or Bmax values, were also similar: $630,000 \pm 280,000$ versus $460,000 \pm$ 86,000 receptors/cell for cells expressing CX3CR1-WT carried out a kinetic study of FKN binding at $37^{\circ} \mathrm{C}$ using the same cell lines tested in Figure 1. Untransfected HEK 293 cells bound extremely low levels of ${ }^{125}$ I-FKN ( $<1 \%$ of radioactivity added) even after 2 hours of incubation, whereas cells expressing CX3CR1-WT reached 50\% of maximal ${ }^{125}$ I-FKN binding by $4.7 \pm 1.7$ minutes (Figure 1c). In contrast, CX3CR1-M280 binding of ${ }^{125}$ I-FKN was markedly and consistently delayed, with $50 \%$ maximal ligand binding occurring at $14.0 \pm 4.5$ minutes, a threefold increase. Once binding had reached equilibrium, labeled FKN was displaced by a 1,000-fold molar excess of unlabeled FKN at a similar slow rate in both CX3CR1-WT and CX3CR1-M280 cells (Figure 1d).

CX3CR1-M280 is defective in cell adhesive function. To test the significance of delayed ligand binding by CX3CR1-M280, we analyzed its adhesive activity using a parallel plate flow chamber and conditions of physiologic shear to model leukocyte-endothelial cell adhesion. Stable K562 cell transfectants were prepared that expressed CX3CR1-WT and CX3CR1-M280 on the surface at equivalent levels, as determined by FACS to 926 or $926-\mathrm{FKN}$ endothelial cell monolayers on glass coverslips at $37^{\circ} \mathrm{C}$ at 0.25 dynes $/ \mathrm{cm}^{2}$ for $5 \mathrm{~min}$ utes. After the initial loading period, the cells were subjected to increasing shear stress; the number of cells remaining adherent after 5 minutes at 10 dynes $/ \mathrm{cm}^{2}$ was quantified. Untransfected K562 cells do not normally express endogenous CX3CR1 and fail to adhere to $926-F K N$ cells (Figure $2 \mathrm{~b}$ ). As previously reported, CX3CR1-WT-expressing cells bound specifically and robustly to $926-\mathrm{FKN}$ cells, but bound at exceedingly low levels to 926 cells lacking FKN (<2\% binding to 926-FKN cells) (16). In three independent studies at $37^{\circ} \mathrm{C}$, cells expressing CX3CR1-M280 showed markedly reduced binding $(28 \% \pm 8.7 \%$ that of CX3CR1-WT) to the 926-FKN monolayer (Figure 2b). Similar results were obtained using FKN-coated glass using mAb 2A9 (Figure 2a). Cells were allowed to bind 

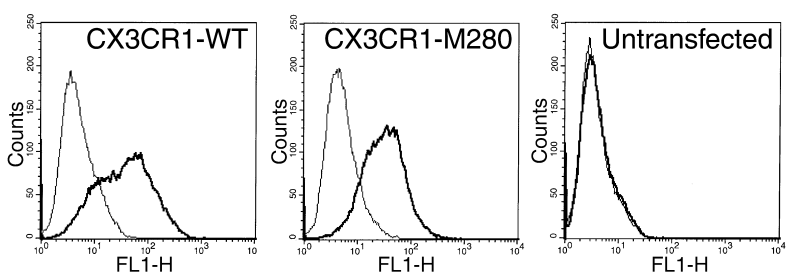

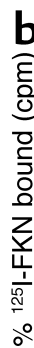

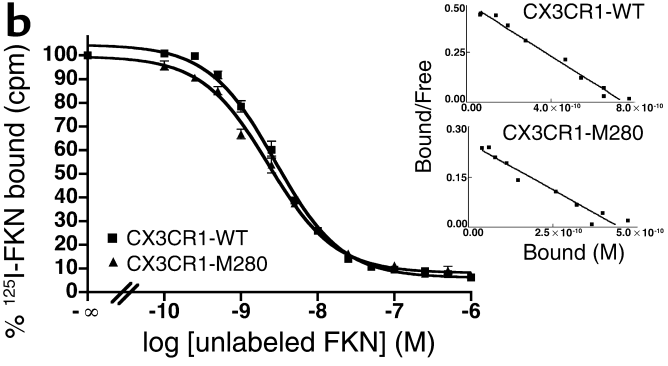

है
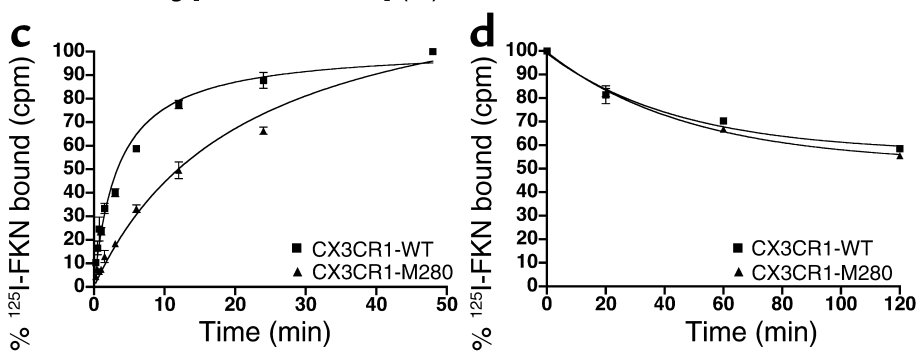

Figure 1

CX3CR1-M280 binds FKN with delayed kinetics. (a) Receptor expression on transfected HEK 293 cell lines. The name of the transfected receptor is at the top of each FACS plot. Thick lines: FITC-conjugated CX3CR1-specific mAb 2A9. Thin lines: isotype antibody controls. Data are representative of three independent experiments. (b) Equilibrium competition binding of $125 \mathrm{I}-\mathrm{FKN}$ to recombinant CX3CR1 variants. Cell lines analyzed in a were incubated with $0.25 \mathrm{nM}^{125} \mathrm{I}-\mathrm{FKN}$ in the presence of azide and the indicated amount of unlabeled FKN for 2 hours at $37^{\circ} \mathrm{C}$. Inset: Scatchard analysis of the binding data. (c) Kinetic binding of ${ }^{125}$ I-FKN to recombinant CX3CR 1 variants. Cell lines analyzed in a were incubated with $0.25 \mathrm{nM}^{125}$ I-FKN at $37^{\circ} \mathrm{C}$ in the presence of azide for the indicated time period. Data in $\mathbf{a}$ and $\mathbf{b}$ are presented as mean \pm SEM and are representative of three independent experiments. (d) Kinetic binding of ${ }^{125} \mathrm{I}-\mathrm{FKN}$ to recombinant CX3CR1 variants. Cell lines analyzed in c were incubated with ${ }^{125}$ I-FKN for 48 minutes at $37^{\circ} \mathrm{C}$, at which time a 1,000 -fold molar excess of unlabeled FKN was added. Serial samples of cells were then removed at the indicated timepoints after addition of the unlabeled ligand, and radioactivity was counted. Data are presented as mean \pm SEM and are representative of three independent experiments. slides (data not shown). The defect was even more pronounced when the analysis was carried out at room temperature (Figure $2 \mathrm{~b}$ ).

Impaired signaling by CX3CR1-M280. In addition to the cell-cell adhesive function mediated by full-length membrane-bound FKN, a chemotactically active soluble form has also been described that acts at CX3CR1 through the classic $\mathrm{G}_{\mathrm{i}}$-coupled pathway common to almost all chemokines, which includes calcium flux. We therefore tested the ability of the recombinant chemokine domain of human FKN to induce calcium flux in the same cell lines assessed for binding activity (Figure 3). Untransfected HEK 293 cells failed to respond to FKN at the highest concentration tested, $1,000 \mathrm{nM}$. In contrast, cells expressing either CX3CR1-WT or CX3CR1-M280 both responded to FKN with kinetics typical for calcium flux responses to chemoattractants; however, the peak

\section{Figure 2}

CX3CR1-M280 has defective adhesion activity. (a) Receptor expression in transfected $\mathrm{K} 562$ cell lines. The name of the transfected receptor is at the top of each FACS plot. Thick lines: CX3CR1-specific mAb 2A9. Thin lines: isotype antibody control. Data are representative of three independent experiments. (b) Flow-based adhesion assay. K562 cells were perfused over monolayers of untransfected (shown as 926) and FKN-transfected (shown as 926FKN) human endothelial cells at room temperature (RT) or $37^{\circ} \mathrm{C}$ and allowed to capture at a shear stress of $0.25 \mathrm{dynes} / \mathrm{cm}^{2}$, then washed at progressively higher shear stresses up to $10 \mathrm{dynes} / \mathrm{cm}^{2}$. The numbers of cells remaining firmly bound to 926 cell monolayers at 10 dynes $/ \mathrm{cm}^{2}$ are shown. Data represent the mean \pm SD of results from three separate experiments at $37^{\circ} \mathrm{C}$ and four separate experiments at room temperature. response was reduced by $50-80 \%$, depending on the concentration tested in cells expressing CX3CR1-M280 compared with cells expressing CX3CR1-WT (Figure 3b).

To test whether native CX3CR1-M280 expressed in primary cells is also defective, we isolated PBMCs from individuals with the appropriate genotypes. PBMCs from two healthy donors homozygous for CX3CR1-M280 exhibited a reduction of approximately $50 \%$ in calcium flux response to FKN at 10, 100,
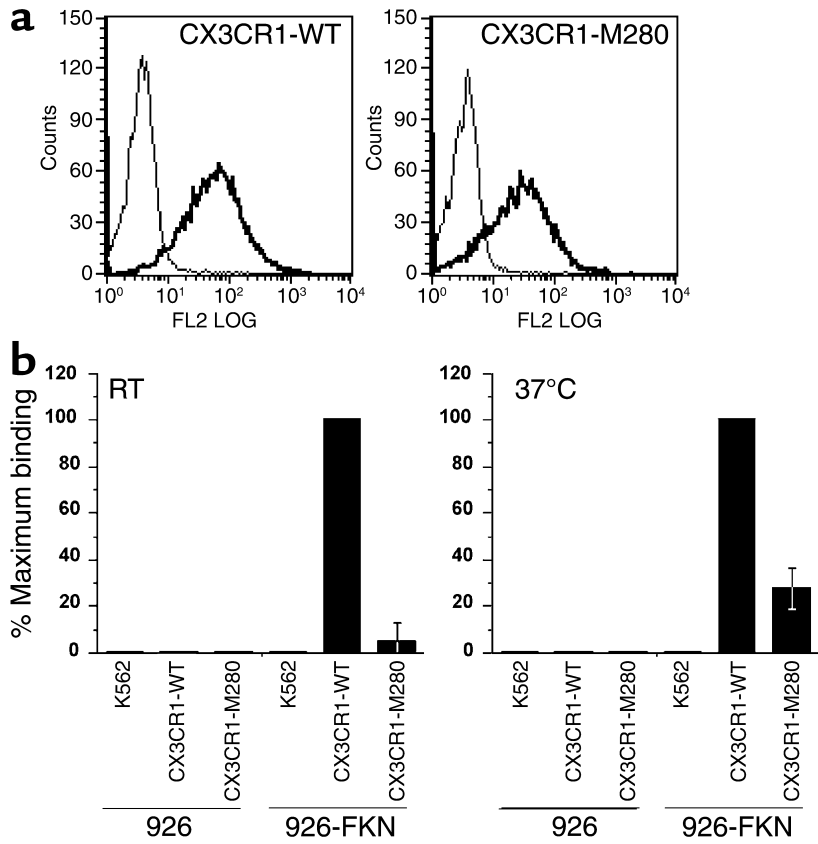

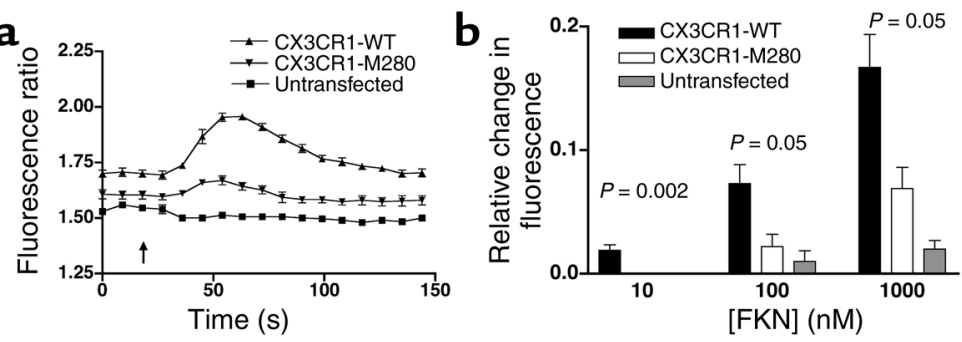

\section{Figure 3}

Calcium mobilization by CX3CR1-M280 is defective. Data represent the mean \pm SEM of one representative experiment (a) and three independent experiments (b). The cell lines used were the same as those analyzed in Figure 1. (a) Kinetics. Shown are realtime fura-2 AM fluorescence traces of untransfected HEK 293 cells or HEK 293 cells expressing the recombinant receptor indicated by the key at upper right in response to $1 \mu \mathrm{M}$ soluble FKN added at 19 seconds. (b) Concentration dependence. Shown is the maximal fura-2 AM fluorescence change of HEK 293 cells stably transfected with the indicated construct in response to the indicated concentration of FKN. $P$ values at the top of each set of bars compare results for CX3CR1-M280 and CX3CR1-WT by two-tailed Student $t$ test.

and 1,000 nM relative to PBMCs from three healthy age- and race-matched donors homozygous for CX3CR1-WT (Figure 4, a and b). The difference appeared to be specific since ATP induced equivalent responses in both groups and the cells showed similar CX3CR1 expression in terms of the percentage of positive cells and mean fluorescence $(<20 \%$ difference, $P>0.15$, data not shown). FKN also induced approximately twofold greater chemotaxis of $\mathrm{CD} 56^{+}$ cells from these same three donors compared with the two CX3CR1-M280 homozygotes (Figure 4c). The difference appeared to be specific since the chemokine SDF-1 (CXCL12), which acts at CXCR4, induced similar chemotactic responses for $\mathrm{CD}^{2} 6^{+}$cells from both groups. CD56 ${ }^{+}$cells from both groups were $90 \%$
$\mathrm{mAb} 2 \mathrm{~A} 9$-positive and the mean fluorescence intensities differed by less than $20 \%(P>0.30)$.

CX3CR1-M280 in the FHS Offspring Cohort. We next tested the epidemiologic association of CX3CR1-M280 with CVD in the FHS Offspring Cohort. As expected, established risk factors and CVD therapy were significantly higher in the CVD group (Table 1). In addition, the observed allele frequencies and compound genotype frequencies of CX3CR1-V249I and CX3CR1T280M polymorphisms in the unstratified FHS Offspring Cohort were similar to those expected based on our previous study of 176 North American Caucasian random blood donors (Table 2) (36). Also consistent with our previous studies, we found that in the present cohort these polymorphisms are in complete linkage disequilibrium (18, 19, 35, 36). Accordingly, of the four possible haplotypes, only three are found $\left(\mathrm{V}^{249} \mathrm{~T}^{280}, \mathrm{I}^{249} \mathrm{~T}^{280}\right.$, and $\left.\mathrm{I}^{249} \mathrm{M}^{280}\right)$. It is important to note for what follows that while M280 is never found in the absence of $I 249$, the converse is not true. In fact, approximately $40 \%$ of haplotypes containing I249 lack M280. Linkage disequilibrium also results in the production of just six of the nine possible compound genotypes (18, $19,35,36)$. The proportion of genotypes for each allele considered separately was consistent with Hardy-Weinberg predictions. Thus, in the absence of stratification by clinical group, the overall genetic makeup of the FHS Offspring Cohort based on polymorphism of CX3CR1 did not deviate from expectations.

Since the defective receptor CX3CR1-M280 actually has two amino acid changes, the functional defect
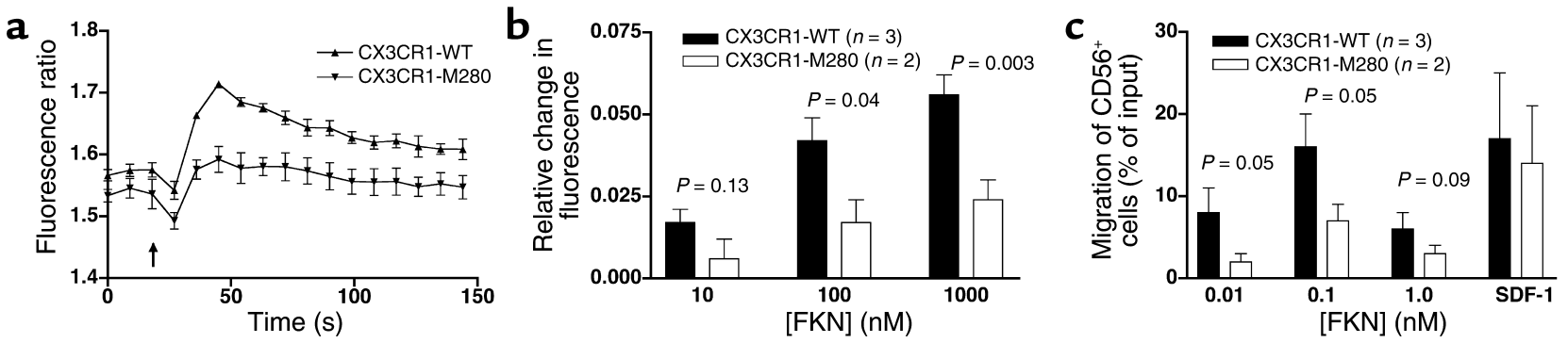

Figure 4

Leukocytes from CX3CR1-M280 homozygotes have defective calcium flux and chemotactic responses to FKN. PBMCs from donors homozygous for CX3CR1-WT (i.e., genotype 1 in Table 2) and CX3CR1-M280 (i.e., genotype 3 in Table 2) were used. (a) Calcium flux: kinetics. Shown are real-time fura- $2 \mathrm{AM}$ fluorescence traces in response to the addition of $1 \mu \mathrm{M}$ soluble FKN (arrow). Data represent the mean $\pm \mathrm{SEM}$ of triplicates, and are representative of four independent experiments. (b) Calcium flux: FKN concentration dependence. Shown is the maximal fura-2 AM fluorescence change of PBMCs in response to the indicated concentration ofFKN. Data are summarized as the mean \pm SEM of results from four independent experiments, two for each CX3CR1-M280 homozygote, in which each condition was tested in triplicate. $P$ values at the top of each set of bars compare results for CX3CR1-M280 and CX3CR1-WT by two-tailed $t$ test. (c) Chemotaxis. PBMCs from the indicated donors were added to the top of Transwell chemotaxis chambers. Specific chemotaxis of CD56 ${ }^{+}$NK cells ( $>90 \%$ mAb 2 A9-positive for all five donors) was determined in response to the indicated concentration of FKN or $10 \mathrm{nM} \mathrm{SDF-1} \mathrm{(CXCL12).} \mathrm{Data} \mathrm{are} \mathrm{summarized} \mathrm{as} \mathrm{the} \mathrm{mean} \pm$ SEM of results from four independent experiments, two for each CX3CR1-M280 homozygote, in which each condition was tested in triplicate. $P$ values at the top of each set of bars compare results for CX3CR1-M280 and CX3CR1-WT by two-tailed Student $t$ test. 
Table 2

Prevalence of CVD according to CX3CR1 genotype

\begin{tabular}{|c|c|c|c|c|c|c|c|c|}
\hline \multirow[t]{2}{*}{ Genotype } & \multicolumn{2}{|c|}{ Amino acid at } & \multirow{2}{*}{$\begin{array}{l}\text { CVD } \\
n(\%)\end{array}$} & \multirow{2}{*}{$\begin{array}{c}\text { No CVD } \\
n(\%)\end{array}$} & \multicolumn{2}{|c|}{ Univariate } & \multicolumn{2}{|c|}{ Adj. } \\
\hline & codon 249 & codon 280 & & & OR & $P$ & OR & $P$ \\
\hline 1 & $\mathrm{~V} / \mathrm{V}$ & $\mathrm{T} / \mathrm{T}$ & $105(51)$ & $794(48)$ & 1.0 & - & 1.0 & - \\
\hline 2 & $1 / 1$ & $\mathrm{~T} / \mathrm{T}$ & $3(1)$ & $23(1)$ & NE & - & - & - \\
\hline 3 & $1 / 1$ & $\mathrm{M} / \mathrm{M}$ & $4(2)$ & $51(3)$ & $\mathrm{NE}$ & - & - & - \\
\hline 4 & $1 / 1$ & $\mathrm{~T} / \mathrm{M}$ & $4(2)$ & $52(3)$ & $\mathrm{NE}$ & - & - & - \\
\hline 5 & $\mathrm{~V} / \mathrm{I}$ & $\mathrm{T} / \mathrm{T}$ & $44(22)$ & $285(17)$ & 1.2 & 0.42 & 1.5 & 0.06 \\
\hline 6 & $\mathrm{~V} / \mathrm{I}$ & $\mathrm{T} / \mathrm{M}$ & $44(22)$ & $450(27)$ & 0.74 & 0.11 & 0.66 & 0.06 \\
\hline $2-6$ & $\mathrm{~V} / \mathrm{I}$ or $\mathrm{l} / \mathrm{I}$ & & $99(49)$ & $861(52)$ & 0.87 & 0.34 & 0.94 & 0.71 \\
\hline $3,4,6$ & & $\mathrm{~T} / \mathrm{M}$ or $\mathrm{M} / \mathrm{M}$ & $52(25)$ & $553(33)$ & 0.68 & 0.02 & 0.60 & 0.008 \\
\hline Total & & & $204(11)$ & $1,655(89)$ & & & & \\
\hline
\end{tabular}

All OR and $P$ values are given for the comparison with genotype 1 except the last, which is with genotypes 1, 2, and 5 (T280 homozygotes); Adj., adjusted in multivariable analysis; NE, not evaluable because of small numbers of participants with these genotypes. $\mathrm{V}$, valine; $\mathrm{T}$, threonine; I, isoleucine; M, methionine.

could be caused by CX3CR1-M280 or CX3CR1-I249 acting alone, or by both acting together. Since CX3CR1M280 has not been observed in the absence of CX3CR1I249 in our studies, its association with a clinical outcome in an epidemiologic study cannot be evaluated separately from CX3CR1-I249. In addition, since only $11 \%$ of the entire cohort has CVD, we calculated that the cohort lacks power at the level of approximately 50\% risk reduction observed in the ACABI and NIH cohort studies to analyze homozygosity of CX3CR1-I249 and CX3CR1-M280 individually, as they represent only $7 \%$ and $3 \%$ of the cohort, respectively. Therefore, as with the ACABI and NIH cohort studies, we analyzed the association of CVD in the FHS Offspring Cohort with CX3CR1-M280 and CX3CR1-I249 individually by dominant (homozygotes plus heterozygotes) genetic models $(18,19)$.

In this cohort, possession of a CX3CR1-M280 allele was less frequent in the CVD group than in those without CVD (25.5\% vs. $33.4 \%$; univariate or unadjusted OR, $0.68 ; 95 \%$ confidence interval, $0.49-0.95 ; P=0.023$, Table 2), suggesting that this allele may act as a protective genetic factor. Importantly, there were no significant differences in any established risk factors when the cohort was stratified by the presence or absence of a CX3CR1-M280 allele (Table 3). Moreover, after multivariable adjustment, possession of CX3CR1-M280 still independently predicted a lower risk of CVD (OR, 0.60; $95 \%$ confidence interval, $0.41-0.88 ; P=0.008)$. In tests for interaction, there were no significant differences in ORs across strata of age, sex, diabetes mellitus, cigarette smoking, hypertension, or use of statin or $\beta$-blocker drugs. The multivariable OR was stronger in men (OR, 0.53 ; confidence interval, $0.32-0.88 ; P=0.015)$ than in women (OR, 0.71; confidence interval, 0.38-1.33; $P=0.28$ ), but the protective association was suggested in both sexes. Three of the six compound genotypes ( 3 , 4 , and 6 in Table 2) contain CX3CR1-M280; however, as mentioned previously, the cohort lacks power to evaluate the first two individually. Thus results for the dominant genetic model for CX3CR1-M280 are largely due to genotype 6 (Table 2).
There was no significant association for the CX3CR1I249 allele in the dominant genetic model in either unadjusted (OR, $0.87 ; P=0.34$ ) or multivariable adjusted (OR, 0.94; $P=0.71$; Table 2) analyses of prevalent CVD (comparing CX3CR1-I249 heterozygotes plus homozygotes versus CX3CR1-V249 homozygotes, i.e., compound genotypes $2-6$ versus compound genotype 1; Table 2). Again, the cohort lacks power to analyze compound genotypes 2, 3, and 4 separately. However, there is sufficient power to analyze compound genotypes 5 and 6 separately versus compound genotype 1 . We have already indicated that compound genotype 6 is associated with reduced risk of CVD; therefore, compound genotype 5 must logically be associated with increased risk of CVD in this cohort, as shown in Table 2. This result was not expected since genotype 5 showed a trend toward decreased risk of CVD in the NIH and ACABI cohort studies (Table 4). This may relate to differences in the design of the studies as well as demographic differences among the recruited patient groups. For example, both the ACABI and NIH cohorts were younger (mean age, 48 years and 57 years, respectively) and more heavily male dominated (male sex, $89 \%$ and $64 \%$, respectively) than the current study cohort. The comparison of genotypes 5 and 6 in the FHS Offspring Cohort is also significant before (OR, 1.6; $P=0.04)$ and after adjustment (OR, 2.3; $P=0.002)$. Since genotype 6 differs from genotype 5 only by heterozygosity for CX3CR1-M280, this suggests that any hazardous effect of CX3CR1-I249 in the FHS Offspring Cohort is reversed by possession of the CX3CR1-M280 allele.

To address the specificity of CX3CR1-M280 association with CVD, we tested three other common polymorphisms: CCR2-64I and CCR5 432 , which are chemokine receptor polymorphisms unlinked to CX3CR1-M280 in genes clustered with CX3CR1 on chromosome 3 p21 $(18,35-37)$, and $S D F 1-3{ }^{\prime} \alpha$, a polymorphism in the gene for the chemokine SDF-1, which is located on chromosome 10 (38). Unlike CX3CR1-M280, none of these exhibited a statistically significant association with CVD in dominant genetic models either before (OR: $0.85,1.14$, and $0.95 ; P=0.42,0.47$, and 0.73 , 
Table 3

Conventional risk factors according to CX3CR1 genotype
Risk factor

Mean age $(\mathrm{yr})$

Male sex (\%)

Diabetes (\%)

Current or former smokers (\%)

Alcohol consumption (oz/wk)

BMI $\left(\mathrm{kg} / \mathrm{m}^{2}\right)$

HDL cholesterol ( $\mathrm{mg} / \mathrm{dl})$

Total cholesterol $(\mathrm{mg} / \mathrm{dl})$

Triglycerides (mg/dl)

Use of statins (\%)

Use of $\beta$ blockers (\%)

Estrogen therapy (\%, women only)

Menopause (\%, women only)
Hypertension (\%)

AP $>0.05$ in all categories. respectively) or after multivariate analysis (OR: 0.70, 1.23 , and $0.99 ; P=0.12,0.32$, and 0.94 , respectively).

\section{Discussion}

We have shown that CX3CR1-M280, a mutant form of the chemokine receptor CX3CR1, is defective in adhesive and chemotactic activity in vitro and is associated with lower risk of CVD in the FHS Offspring Cohort. The latter result, obtained using a population-based, prospective cohort, is consistent with our previous two case-control studies that linked this mutation to reduced risk of atherosclerosis, acute coronary events, and coronary endothelial cell dysfunction $(18,19)$. The association is independent of other CVD risk factors and is similar in magnitude in all three cohorts (Table 4). Moreover, in all three cohorts, the magnitude of risk reduction associated with possession of CX3CR1-M280 is similar to the magnitude of risk augmentation attributable to established CVD risk factors. For example, in the FHS Offspring Cohort, the multivariable adjusted ORs were 2.05 for diabetes, 2.35 for smoking, 1.64 for male sex, and 1.97 for every decade of age, compared with 1.66 (the reciprocal of 0.60 , the OR for CX3CR1-M280 in this cohort) (Tables 1, 2, and 4 and refs. $18,19)$. It is important to emphasize that this level of risk reduction associated with possession of CX3CR1-M280 was observed in a dominant genetic model, indicating that it is due mainly to contributions from heterozygous individuals. Together these data suggest a pathogenic role for CX3CR1 in atherosclerotic CVD in humans. This is further supported by the $A p o E^{-/-}$mouse

\section{Table 4}

model of CVD, since Combadiere et al. have recently shown that $\mathrm{CX} 3 \mathrm{CR}^{-/-} \mathrm{ApoE^{-/- }}$ double knockout mice as well as $\mathrm{CX}_{3} \mathrm{CR}^{+/-} \mathrm{ApoE}^{-/-}$have a greater than $50 \%$ reduction in lesion size at 25 weeks (39).

Our data suggest a model of CVD pathogenesis in which FKN promotes transmigration of CX3CR1 $1^{+}$ leukocytes from the blood into atherosclerotic coronary arteries and their reorganization in plaque. This model is consistent with the prevailing view that atherosclerosis is an inflammatory disease and with the known proinflammatory roles of FKN and CX3CR1 for example as reported in rodent models of cardiac allograft rejection, stroke, and glomerulonephritis (4, 40-44). It is also consistent with the physical presence of both FKN and CX3CR1 in human atherosclerotic plaque (12). FKN is not found in normal human coronary arteries, but has been detected by immunohistochemistry in the intima, media, and adventitia of native atherosclerotic vessels as well as in coronary arteries from cardiac transplants that have developed accelerated atherosclerosis (12). Lesional cells that stain positive colocalize with markers for macrophages, foam cells, and T cells (12). FKN could promote adhesion of these cells to each other (since they appear to also express CX3CR1) and to CX3CR $1^{+}$monocytes transmigrating from the blood into the vessel wall. In this regard, FKN-positive endothelium has been detected in atherosclerotic plaques from coronary arteries in rejected human cardiac transplants, suggesting a potential role for FKN in the initial adhesive interaction of vessel wall with blood leukocytes (12). However, FKN has not been unequivocally detected on endothelium in native atherosclerosis at autopsy $(11,12)$. Lesions found in the latter setting are older and more mature than in transplantation, where a more accelerated form of atherosclerosis occurs. FKN has been found on smooth muscle cells in human atherosclerotic plaques;
Comparison of three cohorts and results for risk of CVD by CX3CR1 genotype using dominant genetic models

\begin{tabular}{|c|c|c|c|}
\hline & $\mathrm{ACABI}$ & $\mathrm{NIH}$ & FHS \\
\hline Type of cohort & Case-Control & Case-Control & Population base \\
\hline Race of participants & Caucasian & Caucasian & Caucasian \\
\hline Location & Paris, France & $\begin{array}{c}\text { Bethesda, } \\
\text { Maryland, USA }\end{array}$ & $\begin{array}{c}\text { Framingham, } \\
\text { Massachusetts, US }\end{array}$ \\
\hline Endpoint & UA, MI & $50 \%$ stenosis & CVD \\
\hline Cases $(n)$ & 151 & 197 & 204 \\
\hline Controls $(n)$ & 249 & 142 & 1,655 \\
\hline Male sex (\%) & 89 & 63 & 50 \\
\hline \multicolumn{4}{|c|}{ Association with $C X 3 C R 1$ alleles: } \\
\hline \multicolumn{4}{|l|}{ I249 dominant: } \\
\hline Unadjusted OR $(P)$ & $0.57(0.008)$ & $0.60(0.02)$ & $0.87(0.34)$ \\
\hline Adjusted OR $(P)$ & $0.43(0.001)$ & $0.54(0.03)$ & $0.94(0.71)$ \\
\hline \multicolumn{4}{|l|}{ M280 dominant: } \\
\hline Unadjusted OR $(P)$ & $0.58(0.03)$ & $0.58(0.02)$ & $0.68(0.02)$ \\
\hline Adjusted OR $(P)$ & $0.49(0.002)$ & $0.58(0.03)$ & $0.60(0.008)$ \\
\hline
\end{tabular}

The ACABI and NIH cohorts are described in more detail in the text and in refs. 18, 19. UA, unstable angina; MI, myocardial infarction; FHS, FHS Offspring. 
however, FKN actions on smooth muscle cells have not yet been delineated (12). At the histological level, CX3CR1 expression parallels that of FKN in atherosclerotic coronary arteries, but unlike FKN it is also diffusely expressed in normal coronary arteries, including on smooth muscle cells (12). Colocalization of FKN and CX3CR1 has not yet been demonstrated within the same cell in atherosclerotic plaque (12).

In this model of pathogenesis, FKN/CX3CR1 interaction may play several roles, but it is clearly not the only factor involved in lesion formation. A protective effect of CX3CR1-M280 may be most pronounced at the level of blood monocyte binding to coronary endothelium, since here the receptor's ligand-binding rate would be most vulnerable to vascular shear force.

Since CX3CR1-M280 allele distribution occurs in Hardy-Weinberg equilibrium, this mutation does not have an overall effect on reproductive fitness. Moreover, we were able to evaluate two individuals who are homozygous for this mutation, both Caucasian men apparently in good health in their late 40s. Thus severe loss of CX3CR1 adhesive function does not appear to be deleterious in humans. This is consistent with the lack of spontaneous disease in CX3CR1 knockout mice and is reminiscent of CCR5 $\Delta 32$, a complete loss-offunction mutation that inactivates the chemokine receptor and major HIV coreceptor CCR5 $(39,45,46)$.

Like any genetic association study, it is possible that the association reported here is due to population stratification. However, this is a population-based study using Caucasians from a single town (Framingham, Massachusetts, USA) where there is relative genetic homogeneity. Also we do not find any statistically significant association with three other common polymorphisms with similar power: CCR2-64I, CCR5 432 , and $S D F 1-3^{\prime} \alpha$, which argues against cryptic population structure explaining the association. Finally, this is the third study demonstrating an association of similar magnitude using three very geographically disparate Caucasian populations (Table 4 ). While it remains possible that the association is due to linkage with another as-yet-unknown polymorphism, evidence that CX3CR1M280 has impaired function and that CX3CR1 knockout mice have reduced susceptibility to atherosclerosis strongly suggests that the association is direct.

Our data identify a series of important new directions for future research. Since the CX3CR1-M280 receptor contains two amino acid changes, the loss of function associated with this receptor may be due to only one or to both, and site-directed mutational analysis will be needed to determine this. Since the CX3CR1-M280 but not the CX3CR1-I249 allele is consistently associated with reduced risk of CVD in three cohorts, we speculate that CX3CR1-M280 is the most likely cause of the functional defect. In addition, CX3CR1-M280 may be generally useful as a genetic probe to evaluate the potential role of CX3CR1 in the pathogenesis of other inflammatory diseases in humans. Reasonable candidates include glomerulonephritis, cardiac transplant rejection, and stroke, all of which have been shown to be ameliorated by genetic activation or immunoneutralization of CX3CR1 or FKN in rodent models $(40-42,44)$.

The chemokine receptors CXCR2 and CCR2 and the CCR2 ligand MCP-1 (CCL2) have also been implicated in atherosclerosis based on mouse models (13-15, 47). A polymorphism of the MCP-1 promoter $(-2,518$ $\mathrm{G} / \mathrm{G})$ that increases promoter activity in vitro has been associated with increased risk of CVD (48). Moreover, the CCR2-64I polymorphism has been associated with reduced coronary artery calcification; however, evidence that CCR2-64I affects receptor function is currently lacking (49). Two studies have linked the CCR5 432 allele with a decreased risk of CVD $(48,50)$. Since CX3CR1-M280 is not linked to these polymorphisms, additional work in larger cohorts will be needed to test whether combinations of these factors further affect risk of CVD. It should also be noted that our current study and another recent Japanese study did not find a statistically significant relationship between these polymorphisms and CVD (51). Consistent with our results in the FHS, this Japanese study also failed to find an association of CX3CR1-I249 with CVD, but CX3CR1-M280 was not tested (51).

In conclusion, our data suggest that the chemokine receptor mutation CX3CR1-M280 in heterozygous form is independently and causally associated with reduced risk of CVD in humans, and that the mechanism may involve reduced inflammatory leukocyte infiltration of the vessel wall. Our results provide support for the inflammation hypothesis of atherosclerosis pathogenesis and identify CX3CR1-M280 as a potential new factor for risk assessment and management in CVD. In addition, our results point to CX3CR1 as a potential new drug target in CVD.

Note added in proof. While this paper was in press, Lesnik et al. (52) reported a similar reduction in atheroscle-

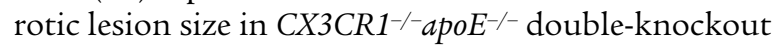
mice to that found in ref. 39 .

1. Desai, M.M., Zhang, P., and Hennessy, C.H. 1999. Surveillance for morbidity and mortality among older adults-United States, 1995-1996. MMWR CDC Surveill. Summ. 48:7-25.2.

2. Wilson, P.W. 1999. Metabolic risk factors for coronary heart disease: current and future prospects. Curr. Opin. Cardiol. 14:176-185.

3. Myers, R.H., Kiely, D.K., Cupples, L.A., and Kannel, W.B. 1990. Parental history is an independent risk factor for coronary artery disease: the Framingham Study. Am. Heart J. 120:963-969.

4. Ross, R. 1999. Atherosclerosis-an inflammatory disease. N. Engl. J. Med. 340:115-126

5. O'Donnell, C.J., and Levy, D. 2000. Weighing the evidence for infection as a risk factor for coronary heart disease. Curr. Cardiol. Rep. 2:280-287.

6. Libby, P., Ridker, P.M., and Maseri, A. 2002. Inflammation and atherosclerosis. Circulation. 105:1135-1143.

7. McDermott, D.H. 2000. Chemokines and their receptors in infectious disease. Springer Semin. Immunopathol. 22:393-415.

8. Murphy, P.M., et al. 2000. International union of pharmacology. XXII. Nomenclature for chemokine receptors. Pharmacol. Rev. 52:145-176.

9. Reape, T.J., and Groot, P.H. 1999. Chemokines and atherosclerosis. Atherosclerosis. 147:213-225.

10. Abi-Younes, S., et al. 2000. The stromal cell-derived factor- 1 chemokine is a potent platelet agonist highly expressed in atherosclerotic plaques. Circ. Res. 86:131-138.

11. Greaves, D.R., et al. 2001. Linked chromosome $16 \mathrm{q} 13$ chemokines, 
macrophage-derived chemokine, fractalkine, and thymus- and activation-regulated chemokine, are expressed in human atherosclerotic lesions. Arterioscler. Thromb. Vasc. Biol. 21:923-929.

12. Wong, B.W., Wong, D., and McManus, B.M. 2002. Characterization of fractalkine (CX3CL1) and CX3CR1 in human coronary arteries with native atherosclerosis, diabetes mellitus, and transplant vascular disease. Cardiovasc. Pathol. 11:332-338.

13. Boring, L., Gosling, J., Cleary, M., and Charo, I.F. 1998. Decreased lesion formation in CCR2-/- mice reveals a role for chemokines in the initiation of atherosclerosis. Nature. 394:894-897.

14. Gu, L., et al. 1998. Absence of monocyte chemoattractant protein-1 reduces atherosclerosis in low density lipoprotein receptor-deficient mice. Mol. Cell. 2:275-281.

15. Dawson, T.C., Kuziel, W.A., Osahar, T.A., and Maeda, N. 1999. Absence of CC chemokine receptor- 2 reduces atherosclerosis in apolipoprotein E-deficient mice. Atherosclerosis. 143:205-211.

16. Fong, A.M., et al. 1998. Fractalkine and CX3CR1 mediate a novel mechanism of leukocyte capture, firm adhesion, and activation under physiologic flow. J. Exp. Med. 188:1413-1419.

17. Harrison, J.K., et al. 1999. Inflammatory agents regulate in vivo expression of fractalkine in endothelial cells of the rat heart. J. Leukoc. Biol. 66:937-944.

18. McDermott, D.H., et al. 2001. Association between polymorphism in the chemokine receptor CX3CR1 and coronary vascular endothelial dysfunction and atherosclerosis. Circ. Res. 89:401-407.

19. Moatti, D., et al. 2001. Polymorphism in the fractalkine receptor CX3CR1 as a genetic risk factor for coronary artery disease. Blood. 97:1925-1928

20. Bazan, J.F., et al. 1997. A new class of membrane-bound chemokine with a CX3C motif. Nature. 385:640-644.

21. Pan, Y., et al. 1997. Neurotactin, a membrane-anchored chemokine upregulated in brain inflammation [erratum 1997, 389:100]. Nature. 387:611-617.

22. Imai, T., et al. 1997. Identification and molecular characterization of fractalkine receptor CX3CR1, which mediates both leukocyte migration and adhesion. Cell. 91:521-530.

23. Haskell, C.A., Cleary, M.D., and Charo, I.F. 1999. Molecular uncoupling of fractalkine-mediated cell adhesion and signal transduction. Rapid flow arrest of CX3CR1-expressing cells is independent of G-protein activation. J. Biol. Chem. 274:10053-10058.

24. Yoneda, O., et al. 2000. Fractalkine-mediated endothelial cell injury by NK cells. J. Immunol. 164:4055-4062.

25. Garton, K.J., et al. 2001. Tumor necrosis factor-alpha-converting enzyme (ADAM17) mediates the cleavage and shedding of fractalkine (CX3CL1). J. Biol. Chem. 276:37993-38001.

26. Tsou, C.L., Haskell, C.A., and Charo, I.F. 2001. Tumor necrosis factoralpha-converting enzyme mediates the inducible cleavage of fractalkine. J. Biol. Chem. 276:44622-44626.

27. Goda, S., et al. 2000. CX3C-chemokine, fractalkine-enhanced adhesion of THP-1 cells to endothelial cells through integrin-dependent and -independent mechanisms. J. Immunol. 164:4313-4320.

28. Murphy, P.M. 1994. The molecular biology of leukocyte chemoattractant receptors. Annu. Rev. Immunol. 12:593-633.

29. Harrison, J.K., et al. 2001. Mutational analysis of the fractalkine chemokine domain. Basic amino acid residues differentially contribute to CX3CR1 binding, signaling, and cell adhesion. J. Biol. Chem. 276:21632-21641.

30. Campbell, J.J., et al. 1998. 6-C-kine (SLC), a lymphocyte adhesion-triggering chemokine expressed by high endothelium, is an agonist for the MIP-3beta receptor CCR7. J. Cell Biol. 141:1053-1059.

31. Dawber, T.R., and Kannel, W.B. 1966. The Framingham study. An epidemiological approach to coronary heart disease. Circulation. 34:553-555

32. Kannel, W.B., Feinleib, M., McNamara, P.M., Garrison, R.J., and Castelli, W.P. 1979. An investigation of coronary heart disease in families. The
Framingham offspring study. Am. J. Epidemiol. 110:281-290.

33. Kannel, W.B., and Cupples, A. 1988. Epidemiology and risk profile of cardiac failure. Cardiovasc. Drugs Ther. 1(Suppl. 2):387-395.

34. Combadiere, C., et al. 1998. Identification of CX3CR1. A chemotactic receptor for the human $\mathrm{CX} 3 \mathrm{C}$ chemokine fractalkine and a fusion coreceptor for HIV-1. J. Biol. Chem. 273:23799-23804.

35. Faure, S., et al. 2000. Rapid progression to AIDS in HIV+ individuals with a structural variant of the chemokine receptor CX3CR1. Science. 287:2274-2277.

36. McDermott, D.H., et al. 2000. Genetic polymorphism in the HIV coreceptor CX3CR1 and risk of HIV disease. Science. 290:2031a. http://www sciencemag.org/cgi/reprint/290/5499/2031a.pdf.

37. Smith, M.W., et al. 1997. Contrasting genetic influence of CCR2 and CCR5 variants on HIV-1 infection and disease progression. Hemophilia Growth and Development Study (HGDS), Multicenter AIDS Cohort Study (MACS), Multicenter Hemophilia Cohort Study (MHCS), San Francisco City Cohort (SFCC), ALIVE Study. Science. 277:959-965.

38. Winkler, C., et al. 1998. Genetic restriction of AIDS pathogenesis by an SDF-1 chemokine gene variant. ALIVE Study, Hemophilia Growth and Development Study (HGDS), Multicenter AIDS Cohort Study (MACS), Multicenter Hemophilia Cohort Study (MHCS), San Francisco City Cohort (SFCC). Science. 279:389-393.

39. Combadiere, C., et al. 2003. Decreased atherosclerotic lesion formation in CX3CR1/Apolipoprotein E double-knockout mice. Circ. 107:1009-1016.

40. Haskell, C.A., et al. 2001. Targeted deletion of $\mathrm{CX}_{3}$ CR1 reveals a role for fractalkine in cardiac allograft rejection. J. Clin. Invest. 108:679-688. doi:10.1172/JCI200112976.

41. Robinson, L.A., et al. 2000. A role for fractalkine and its receptor CX3CR1) in cardiac allograft rejection. J. Immunol. 165:6067-6072.

42. Soriano, S.G., et al. 2002. Mice deficient in fractalkine are less susceptible to cerebral ischemia-reperfusion injury. J. Neuroimmunol. 125:59-65.

43. Tarozzo, G., Campanella, M., Ghiani, M., Bulfone, A., and Beltramo, M. 2002. Expression of fractalkine and its receptor, CX3CR1, in response to ischaemia-reperfusion brain injury in the rat. Eur. J. Neurosci. 15:1663-1668.

44. Feng, L., et al. 1999. Prevention of crescentic glomerulonephritis by immunoneutralization of the fractalkine receptor CX3CR1 rapid communication. Kidney Int. 56:612-620.

45. Jung, S., et al. 2000. Analysis of fractalkine receptor CX(3)CR1 function by targeted deletion and green fluorescent protein reporter gene insertion. Mol. Cell. Biol. 20:4106-4114.

46. Zimmerman, P.A., et al. 1997. Inherited resistance to HIV-1 conferred by an inactivating mutation in CC chemokine receptor 5: studies in populations with contrasting clinical phenotypes, defined racial background, and quantified risk. Mol. Med. 3:23-36.

47. Boisvert, W.A., Santiago, R., Curtiss, L.K., and Terkeltaub, R.A. 1998. A leukocyte homologue of the IL-8 receptor CXCR- 2 mediates the accumulation of macrophages in atherosclerotic lesions of LDL receptordeficient mice. J. Clin. Invest. 101:353-363.

48. Szalai, C., et al. 2001. Involvement of polymorphisms in the chemokine system in the susceptibility for coronary artery disease (CAD). Coincidence of elevated Lp(a) and MCP-1 -2518 G/G genotype in CAD patients. Atherosclerosis. 158:233-239.

49. Valdes, A.M., et al. 2002. Val64Ile polymorphism in the C-C chemokine receptor 2 is associated with reduced coronary artery calcification. Arterioscler. Thromb. Vasc. Biol. 22:1924-1928.

50. Gonzalez, P., et al. 2001. Genetic variation at the chemokine receptors CCR5/CCR2 in myocardial infarction. Genes Immun. 2:191-195.

51. Yamada, Y., et al. 2002. Prediction of the risk of myocardial infarction from polymorphisms in candidate genes. N. Engl. J. Med. 347:1916-1923.

52. Lesnik, P., Haskell, C.A., and Charo, I.F. 2003. Decreased atherosclerosis in $\mathrm{CX}_{3} \mathrm{CR} 1^{-/-}$mice reveals a role for fractalkine in atherogenesis. J. Clin. Invest. 111:333-340. doi:10.1172/JCI200315555. 